\title{
Adaptive Multicriteria Thresholding for Cooperative Spectrum Sensing in Cognitive Radio Ad Hoc Smart Grid Networks under Shadowing Effect
}

\author{
Kanabadee Srisomboon, Yutthna Sroulsrun and Wilaiporn Lee *(D)
}

check for updates

Citation: Srisomboon, K.; Sroulsrun, Y.; Lee, W. Adaptive Multicriteria Thresholding for Cooperative Spectrum Sensing in Cognitive Radio Ad Hoc Smart Grid Networks under Shadowing Effect. Energies 2021, 14, 2259. https://doi.org/10.3390/ en14082259

Academic Editors: Thomas Strasser, Sebastian Rohjans and Graeme Burt

Received: 19 March 2021

Accepted: 15 April 2021

Published: 17 April 2021

Publisher's Note: MDPI stays neutral with regard to jurisdictional claims in published maps and institutional affiliations.

Copyright: (c) 2021 by the authors. Licensee MDPI, Basel, Switzerland. This article is an open access article distributed under the terms and conditions of the Creative Commons Attribution (CC BY) license (https:// creativecommons.org/licenses/by/ $4.0 /)$.
Department of Electrical and Computer Engineering, Faculty of Engineering, King Mongkut's University of Technology North Bangkok, Bangkok 10800, Thailand; kanabadee.s@eng.kmutnb.ac.th (K.S.); yutthna.s@rmutp.ac.th (Y.S.)

* Correspondence: wilaiporn.1@eng.kmutnb.ac.th

Abstract: Cognitive radio is expected to be implemented in smart grids since it presents high reliability, high accuracy and low transmission time by utilizing licensed bands opportunistically. Shadowing environment affects the performance of channel availability detection of local spectrum sensing since it occurs occasionally. Therefore, the cooperative spectrum sensing is encouraged to be used for addressing shadowing issues. The principle cooperative spectrum sensing techniques suffer from unreliable local information from secondary users (SUs) who are encountered by the shadowing effect. Then, several alternative methods, adaptive majority rule and improved weight algorithm (IMA) is proposed by taking the SUs reliability into account. However, the unreliable SUs are still considered according to the algorithm. Therefore, in this paper, we propose an adaptive multi-criteria thresholding (AMT) to determine the channel availability according to the SUs reliability. The main contribution of AMT is three-fold. First, the new reliable weight calculation is proposed by utilizing analytic hierarchy process (AHP) under three major criteria. Second, AMT is flexible to the number of SUs since it adapts the decision weight on the optimal number of SUs according to the reliable SUs. Third, the shadowing issue is addressed by taking only reliable SUs into account.

Keywords: cooperative spectrum sensing; fusion rule; AHP; shadowing; smart grid

\section{Introduction}

During the last decade, a number of wireless communication applications have noticeably grown together with communication resources demand. Smart grid [1] is the modern electrical grid which comprises of the communication module for exchanging information between utility, data concentration unit (DCU) and customers. Therefore, communication is a critical function of smart grid that has to be reliable, efficient and fast. However, the conventional static communication channel allocation cannot meet the requirement of emerging smart grid technology and others.

Stated by traditional policy, licensed bands cannot be utilized by unlicensed users or a secondary user (SU) even if the licensed bands are not utilized by primary users (PUs). Therefore, the unlicensed spectrum resource cannot satisfy the current spectrum demand. Depending on the current underutilization of licensed spectrum resource, a cognitive radio (CR) [2-7] is encouraged to be used for addressing the underutilization issue. By utilizing CR technology, not only PU can use the licensed band, it can be dynamically utilized by SUs with unharmed interference to the PU. As stated in the first cognitive radio standard, IEEE 802.22 [8], television bands are allowed to be utilized by the SU where two types of PU signal are considered, i.e., TV broadcasting and wireless microphone signal.

In the CR networks, a SU can communicate to others in two ways. First, in infrastructurebased networks, the SU communicates to others via a base station (or fusion center) who gathers the information from SUs for making decisions such as declaring the availability 
of the considered channel. Second, ad hoc networks [9], the SU communicates to others through the neighboring SUs. However, before starting the communication, the SU must ensure that its communication must not interfere with the PU or the communication channel is not being utilized. In practice, the ad-hoc nodes, or SUs, are considered as mobile devices with their location changing dynamically and the received PU signal strength may vary according to several factors including the distance between them and the PU transmitter, varying environment and obstacles in transmission path of the received PU signal. Therefore, the performance of detecting the channel availability of ad-hoc network may suffer from those environments than the infrastructure based. Therefore, we focus on the ad-hoc nodes. Spectrum sensing [10-46] is the vital function of CR which is used to identify the PU's state. To achieve the spectrum usage requirement of $C R$, an efficient spectrum sensing is required. If the spectrum sensing presents a wrong declaration about PU state, the SU will start its communication and will cause interference to the PU.

Principally, non-cooperative spectrum sensing techniques are widely considered where the PU's state is identified by an SU individually. In practice, there are several issues that degrade the spectrum sensing performance including the low quality of PU's signal due to low signal-to-noise ratio (SNR) [11-14], noise uncertainty [14,15], and shadowing effect $[16,17]$. Low SNR and noise uncertainty issues can be addressed by modifying the non-cooperative spectrum sensing techniques [18-34]. Energy detection [19-21] is the simplest non-cooperative technique which compares the detected signal energy to the predetermined threshold in order to identify the PU's state. However, this simple technique gives an inefficient spectrum sensing performance under low SNR and noise uncertainty issues. The covariance-based spectrum sensing [22] was proposed to address the noise uncertainty issue. However, it comes at a cost of high computational burden and long sensing time. Then, a number of solutions of ED were proposed to address these issues, e.g., adaptive schemes for energy detection [23-25], two-stage spectrum sensing [26], multislot techniques [27], spectrum sensing based eigen-decomposition [28,29] and learning based spectrum sensing [30-34]. However, these techniques still identify the PU's state individually and cannot address the issues of shadowing.

Depending on the shadowing issue, the signal of the PU, i.e., PU in an area that the broadcast signal is obstructed by objects, cannot be detected by the SU since the broadcast signal from the PU is obstructed by buildings where the strength of the signal is lower than can be detected, as depicted in Figure 1. Then, the individual spectrum sensing may declare a wrong state of the PU, e.g., the PU occupies the licensed channel but the SU declares the channel as available instead of unavailable affected by the hidden PU. Therefore, a cooperative spectrum sensing is determined as the efficient strategy for addressing the shadowing issue. Once the PU's state is identified cooperatively using local information from several SUs, based on cooperative strategy, the fusion center assigns a number of SUs to sense the PU's state and the detected results are reported to the fusion center via the control channel. The fusion center identifies the PU's state by combining the gathered information using the information fusion rules. Then, the fusion rule is the vital method of cooperative spectrum sensing.

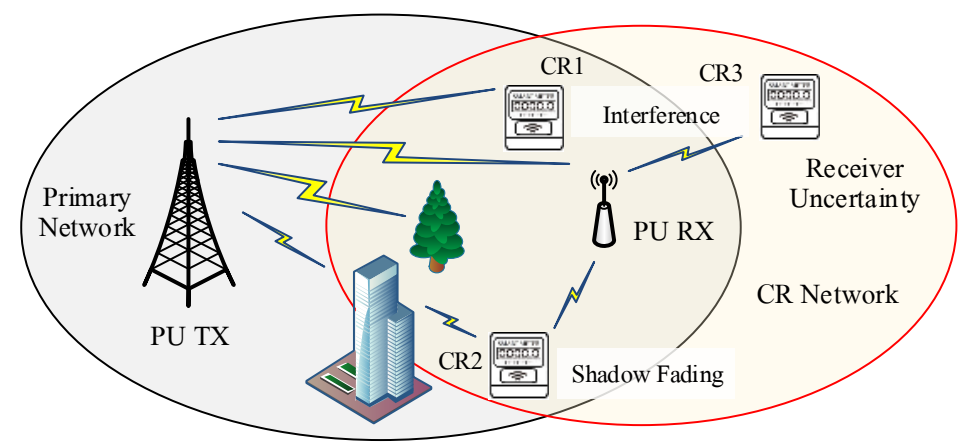

Figure 1. Issue of shadowing effect under smart grid environment. 
In general, there are two data fusion schemes including hard combination and soft combination. For hard combination rules [35-40], the fusion center receives the local binary decision, 0 or 1 , from several SUs and then performs the data fusion via the hard combination rules-OR, AND and majority $[35,36]$. It should be noted that the binary 1 identifies an existent state of PU while binary 0 identifies an inexistent state. Nevertheless, the expected performance is not met by using the traditional information fusion rules due to their merits/demerits. On the other hand, soft combination strategy [41] combines the local sensing data transmitted by several SUs and the fusion center combines this information and constructs the global test statistic. Then, it is compared to the soft decision threshold to identify the PU's state. However, the soft fusion scheme comes at a cost of system complexity and the computational burden. Therefore, the hard combination becomes more popularly utilized and study takes place to improve the performance of principle techniques_-OR, AND and majority rule — through several solutions. It should be mentioned that the performance of spectrum sensing can be evaluated through two vital metrics including cooperative probability of detection $\left(Q_{d}\right)$ and cooperative probability of false alarm $\left(Q_{f}\right) . Q_{d}$ is determined as the correct declaration when the PU exists. $Q_{f}$ is determined as the wrong declaration when the PU does not exist.

The first principal fusion rule, OR rule, declares the channel status as unavailable once a PU is detected by a single SU. Although the OR fusion rule presents a high probability of detection since only a single SU detects the PU existence, it also presents a high false declaration, high $Q_{f}$, since the SU may send the wrong detection result to the fusion center. The second rule, AND rule, declares the channel status as unavailable once all of SUs detect the existence of PU. Even if the AND fusion rule addresses the issue of false declaration of the PU state when the PU does not exist, the correct declaration when the PU exists is also low, i.e., low $Q_{d}$. The third rule, the majority rule, address the issues of OR rule and AND rule by declaring the channel status as unavailable once K SUs detect the PU existence. However, it still cannot achieve the satisfied spectrum sensing performance under low SNR due to path loss and high strength of shadowing effect.

For the majority fusion rule, the number of SUs is considered as the critical factor that affects the performance of the cooperative spectrum sensing. In [42], an improved weight cooperative spectrum sensing (IMA) is proposed by adapting the cooperative threshold on the optimal number of SUs and determines the cooperative decision statistic based on the SUs reliability. The ratio PU detection of each SU as compared to the PU detection of the fusion center is determined as the SU reliability. The optimal number of SUs $[43,44]$ is calculated with the objective to minimize the error rate of cooperative spectrum sensing including $Q_{f}$ and the cooperative missed detection probability $\left(Q_{m}\right)$ where $Q_{m}$ is determined as the wrong declaration when the PU exists. Therefore, the IMA method improves the $Q_{d}$ of the majority rule while it presents low $Q_{f}$. In [45], modified majority rule is proposed by determining the PU state on $K_{R}$ reliable SU. In [46], the number of considered SUs according to the majority rule is proposed by taking the energy of the SUs into account with threshold adapting according to the SUs energy. The reliability of the modified majority rule is considered by analytic hierarchy process (AHP) [47-49] where the past PU detection and the distance between SUs and the PU is the factor to determine the SU reliability. It can be noticed that the reliability of the SU and the optimal number of SU have a great impact on the performance of majority rule and its modified versions. With an efficient cooperative scheme and reliable information from SUs, the fusion center can determine the PU state efficiently. On the other hand, the machine learning algorithms are implemented for cooperative spectrum sensing. In [50], the machine learning algorithms are implemented into cooperative spectrum sensing function and therefore the performance of these techniques is investigated. In [51], the learning-based algorithm is proposed to learn the PU behavior using Q-learning. The algorithm learns the PU behavior using local information from SUs. However, the learning based cooperative spectrum sensing techniques require prior information to learn the behavior of PU to generate the cooperative sensing model. 
In this paper, a novel modified majority rule, adaptive multi-criteria thresholding algorithm (AMT), is proposed by considering the reliability of SUs on multi-criteria and adapting the cooperative spectrum sensing threshold on the optimal number of SUs as corresponding to the reliability weight. Currently, several multi-criteria ranking methods are proposed, e.g., analytic hierarchy process (AHP), technique for order of preference by similarity to ideal solution (TOPSIS) [52], Vise Kriterijumsa Optimizacija I Kompromisno Resenje (VIKOR) [52], complex proportional assessment (COPRAS) [52] and preference ranking organization method for enrichment of evaluations II (PROMETHEE II) [52]. These methods perform decision-making under different frameworks. AHP determines the rank of alternatives in a hierarchical manner where the main criteria and alternative weight are determined by using a pairwise-comparison. TOPSIS and VIKOR determine the rank of alternatives by determining the distance of each alternative according to the ideal positive and negative solutions where the distance of TOPSIS is based on the Euclidean solution while VIKOR is based on linear normalization. COPRAS ranks the alternatives according to maximizing and minimizing criteria through the summation of weighted normalized values. PROMETHEE II determines the ranking on the outranked relationship mechanism.

Although AHP is considered as the conventional multi-criteria ranking method, it presents a robustness and flexibility to perform the performance decision under complex constrains. For other methods, the main criteria weighting is considered as a main issue since it is difficult to assign the weight to each criterion individually. For AHP, the main criteria are compared in a pairwise mechanism, therefore, it is easy to determine the importance between criteria. Even if the number of criteria and alternatives are the limit of $\mathrm{AHP}$, in this paper, our proposed technique, AMT, does not suffer from the limitation since only three main criteria are taken into account with 10 alternatives (SUs). Moreover, AMT benefits from the pairwise comparison mechanism, therefore the ranking weights can be determined effectively.

The contribution of this paper is three-fold. First, the new reliable weight calculation is proposed by utilizing AHP under three major criteria-collective detection rates, collective false detection rate and estimated SNR of detected signal. Therefore, the reliability weight of SUs is generated effectively. Therefore, AMT determines the channel availability according to the reliable SUs. Then, it can achieve the highest $Q_{d}$ as compared to others hard cooperative spectrum sensing and maintains a low $Q_{f}$ as stated by IEEE802.22 protocol where $Q_{f}$ should be less than 0.1 . Second, we propose a new adaptive cooperative threshold scheme which adapts on the optimal number of SUs and their reliability weights. Therefore, AMT is flexible to the number of SUs since it adapts the decision weight on the optimal number of SUs according to the reliable SUs. Third, the shadowing issue is addressed since the information from unreliable SUs is neglected from the decision process.

This paper is organized as follows: the cooperative spectrum sensing including problem statements and existing cooperative spectrum sensing techniques are briefly introduced in Section 2. In Section 3, the proposed technique adaptive multicriteria thresholding algorithm (AMT) is described in detail. Then, the simulation results are depicted and discussed in Section 4. Finally, the conclusion is drawn in Section 5.

\section{Related Works}

In this section, we will discuss our problem statement and describe three conventional cooperative spectrum sensing rules including AND, OR and majority rule.

\subsection{Problem Statement}

In wireless communication environments, many factors such as the noise uncertainty, shadowing and path loss decrease the performance of non-cooperative spectrum sensing. Since the location of SUs are different, therefore, for some SUs, the received signal from the PU may be degraded via transmission path due to obstacles such as buildings. This 
phenomenon is called the shadowing effect $(\sigma)$ that degrades the quality of the received signal. The mathematic model of the shadowing effect $(\sigma)$ [17] can be written as

$$
\sigma=0.1 \ln (10) \sigma_{d B}
$$

where $\sigma_{d B}$ is log-normal shadowing in $d B$. The channel gain can be modeled log-normal shadowing variable $e^{x}$ where $x$ is a zero-mean Gaussian random variable with variance $\sigma^{2}$ [17].

On the other hand, the received signal from the PU suffers from the signal's propagation distance. This is known as path loss effect [12-14] where the mathematical model is expressed as:

$$
P L \equiv C d^{-\aleph}
$$

where $P L$ is path loss, $d$ is distance between PU and SU, $C$ is loss constant and $\aleph$ is path loss exponent.

The hypothesis models of the received signal [4] are given by:

$$
\mathbf{x}=\left\{\begin{array}{lll}
\boldsymbol{\eta} & \text { when PU absents } & {\left[H_{0}\right]} \\
\mathbf{h} *(P L \cdot \mathbf{s})+\boldsymbol{\eta} & \text { when PU presents } & {\left[H_{1}\right]}
\end{array}\right.
$$

where $\mathbf{x}$ is the received signal of $\mathrm{SU}, \boldsymbol{\eta}$ is additive white Gaussian noise, $\mathbf{s}$ is the signal of $\mathrm{PU}$ and $\mathbf{h}$ is gain of channel.

In general, the energy detection is considered as the local spectrum sensing of each $\mathrm{SU}$, therefore the local probability of detection $\left(P_{d}\right)$ and local probability of false alarm $\left(P_{f}\right)[4]$ are given as:

$$
\begin{gathered}
P_{d}=Q\left[\sqrt{\frac{N_{\mathrm{m}}}{2}}\left(\frac{\lambda}{\sigma_{\mathfrak{\eta}}^{2}}-(S N R)-1\right)\right] \\
P_{f}=Q\left[\left(\frac{\lambda}{\sigma_{\eta^{2}}^{2}}-1\right) \sqrt{\frac{N_{\mathrm{m}}}{2}}\right]
\end{gathered}
$$

where $\lambda$ is the decision threshold of the energy detection, $\sigma_{\mathfrak{\eta}}{ }^{2}$ is variance of additive white Gaussian noise and $N_{\mathrm{m}}$ is the signal of the sample.

\subsection{Cooperative Spectrum Sensing}

To combat the shadowing effect, cooperative spectrum sensing (CSS) schemes are proposed. In CSS network, the PU is sensed in a cooperative manner by a number of SUs where the SU shares its detection result to others. For the master/slave mode of ad hoc networks, the fusion center is determined as the master node that senses the PU existence via the gathered detection results from SUs. Then, the final declaration of PU status is performed by the fusion center. There are three traditional fusion rules including OR, AND and majority rule [37-40]. It should be mentioned that $Q_{f}$ is the probability of false alarm and $Q_{d}$ is the probability of detection of cooperative scheme.

\subsubsection{OR Rule}

The fusion center declares the status of channel as occupied when only a single SU detects the existence of the PU. Then, the $Q_{f}$ and $Q_{d}$ [37-40] can be written as:

$$
\begin{aligned}
& Q_{f}=1-\left(1-P_{f}\right)^{N} \\
& Q_{d}=1-\left(1-P_{d}\right)^{N}
\end{aligned}
$$

where $N$ is the number of the SU. 


\subsubsection{AND Rule}

After the fusion center gathers all SUs detection results, it declares the channel status as occupied when all SUs detect the PU. Then, the $Q_{f}$ and $Q_{d}[37-40]$ is given as:

$$
\begin{aligned}
& Q_{f}=\left(P_{f}\right)^{N} \\
& Q_{d}=\left(P_{d}\right)^{N}
\end{aligned}
$$

\subsubsection{Majority Rule}

For majority rule, the fusion center declares the channel status as occupied when at least $k$ from $N$ SUs detect the PU. Then, the $Q_{f}$ and $Q_{d}[37-40]$ is given as:

$$
\begin{gathered}
Q_{f}=\sum_{l=k}^{N}\left(\begin{array}{c}
N \\
l
\end{array}\right)\left(P_{f}\right)^{l}\left(1-P_{f}\right)^{N-l} \\
Q_{d}=\sum_{l=k}^{N}\left(\begin{array}{c}
N \\
l
\end{array}\right)\left(P_{d}\right)^{l}\left(1-P_{d}\right)^{N-l}
\end{gathered}
$$

\subsubsection{Improved Weight Algorithm}

The improved weight cooperative spectrum sensing (IMA) [42] is the modified version of the traditional majority rule. To improve the performance of the majority rule, the reliability of the information taken from the SUs and the optimal number of SUs are taken into account. By determining the total error spectrum sensing rate, the optimal number of $\mathrm{SU}\left(n_{\text {opt }}\right)$ is obtained and can be expressed as

$$
n_{\text {opt }}=\min \left(N, \frac{N}{1+\beta}\right)
$$

where

$$
\beta=\frac{\ln \frac{P_{f}}{1-P_{m}}}{\ln \frac{P_{m}}{1-P_{f}}}
$$

where $P_{m}$ is the local probability of missed detection of each SU.

Moreover, the reliability weight determined by the ratio of PU detection of each SU as compared to the fusion center is taken into account of binary decisions from SUs. Then, the decision statistic of IMA is compared to the IMA threshold which is given by:

$$
\lambda_{\mathrm{IMA}}=\left(\frac{n_{\text {opt }}}{N}\right)
$$

If the cooperative decision statistic of IMA is greater than $\lambda_{\text {IMA }}$, the PU state will be declared as existent. Otherwise, the PU state will be declared as not existent. The cooperative decision statistic of IMA is determined by the linear combination between the weight vector and the local decision vector where the weight vector is according to the collective probability of detection and the local decision vector is the local decision from each SUs.

\subsubsection{Adaptive Majority Rule}

Adaptive majority rule [45] is the alternative method of the majority rule which determines the reliability of SUs through the multi-criteria ranking method, analytic hierarchy process (AHP) [49-51]. For the adaptive majority rule, two metrics are taken into account-past PU detection and the distance between SUs and the PU. Then, the reliability score of each SU is computed through the combination of eigen-decomposition vector of 
actual values of sub-criteria, i.e., past PU detection of each SU and the distance between the $\mathrm{SU}$ and PU, corresponding to the eigen-decomposition vector of main criteria.

Once the reliable SUs are considered, the only SUs with $K_{R}$ highest reliability score are determined. To declare the existence state of PU, at least $\frac{K}{2}$ of SUs with $K_{R}$ highest reliability score must present the binary 1 of its local detection.

\section{Proposed Algorithm: Adaptive Multicriteria Thresholding}

In this section, adaptive multicriteria thresholding algorithm (AMT) is proposed to improve the reliable weight efficient utilizing analytic hierarchy process (AHP). The framework of AMT algorithm consists of two critical modules-reliability weight $\left(\mathrm{W}_{\mathrm{crs}}\right)$ calculation and adaptive AMT thresholding. By taking the advantage of the cooperative spectrum sensing from only reliable users with efficient reliable weight, the issue of shadow PU under low signal-to-noise ratio (SNR) can be addressed. The framework of AMT algorithm is shown in Algorithm 1.

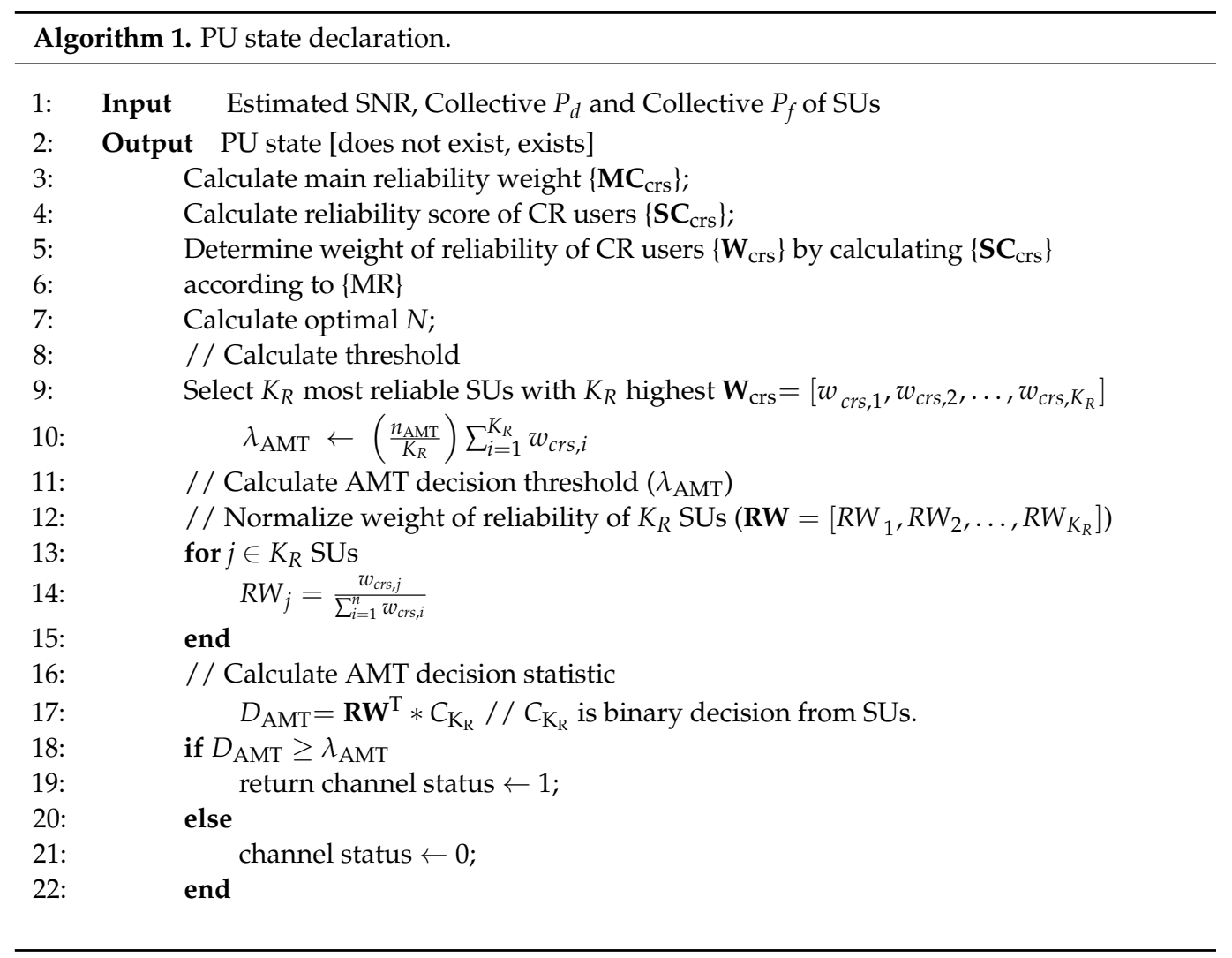

First, by using AHP method, the main criteria $\left(\mathbf{M C}_{\mathrm{crs}}=\left[m c_{1}, m c_{2}, m c_{3}\right]\right)$ is determined through three factors including collective detection rate, collective false detection rate and estimated SNR of detected signal. It should be mentioned that the collective detection rate is determined as the correct detection of each SU as compared to the detection status of the fusion center. On the other hand, collective false detection considered as a wrong detection of each SU as compared to the detection status of the fusion center.

Second, the reliability weight of SUs $\left(\mathbf{W}_{\mathrm{crs}}=\left[w_{c r s, 1}, w_{c r s, 2}, \ldots, w_{c r s, n}\right]\right)$ is computed by computing the sub-criteria weight of each $\mathrm{SU}\left(\mathrm{SC}_{\mathrm{crs}}=\left[s c_{1}, s c_{2}, \ldots, s c_{n}\right]\right)$ corresponding to $\mathbf{M C}_{\text {crs }}$, and only SUs with the highest $K_{R}$ reliability weight are selected. Third, the AMT decision threshold $\left(\lambda_{\mathrm{AMT}}\right)$ and AMT decision statistic $\left(D_{\mathrm{AMT}}\right)$ are computed based on the optimal number of SU and normalized reliable weight (RW). Finally, the PU state is determined by comparing $D_{\mathrm{AMT}}$ to $\lambda_{\mathrm{AMT}}$. 


\subsection{Reliability Weight Calculation}

In this subsection, the reliability weight calculation is described in detail together with the mathematical models. To determine the reliability weight, the AHP method with three vital performance of SU-collective detection rate, collective false detection rate and estimated SNR of detected signal—is determined.

1. Construct the structure of reliability weight calculation based on AHP method as depicted in Figure 2.

2. Construct the pairwise comparison matrix (A) [49] by pairwise comparing each main criterion to others. Then, the $\mathbf{A}$ can be expressed as

$$
\mathbf{A}=\left[\begin{array}{ccc}
1 & a_{12} & a_{13} \\
a_{21} & 1 & a_{23} \\
a_{31} & a_{32} & 1
\end{array}\right]
$$

where $a_{12}$ is the weight of pairwise comparison of collective detection rate to a collective false detection rate, $a_{13}$ is the weight of pairwise comparison of collective detection rate to the estimated SNR of detected signal and $a_{23}$ is the weight of pairwise comparison of collective false detection rate to the estimated SNR of detected signal. It should be noted that $a_{21}=1 / a_{12}, a_{31}=1 / a_{13}$ and $a_{32}=1 / a_{23}$.

3. Calculate main reliability weight $\left(\mathbf{M C}_{\mathrm{crs}}\right)$ by normalizing the calculated eigen-vector of $\mathbf{A}$.

4. Perform procedure 1 to 3 for the sub-criteria of each criterion, then $\mathbf{S C}_{\mathrm{crs}}$ is obtained.

5. Determine the reliability weight of $\mathrm{SUs}\left(\mathbf{W}_{\mathrm{crs}}\right)$ by computing $\mathbf{S C}_{\mathrm{crs}}$ as corresponding to $\mathrm{MC}_{\mathrm{crs}}$.

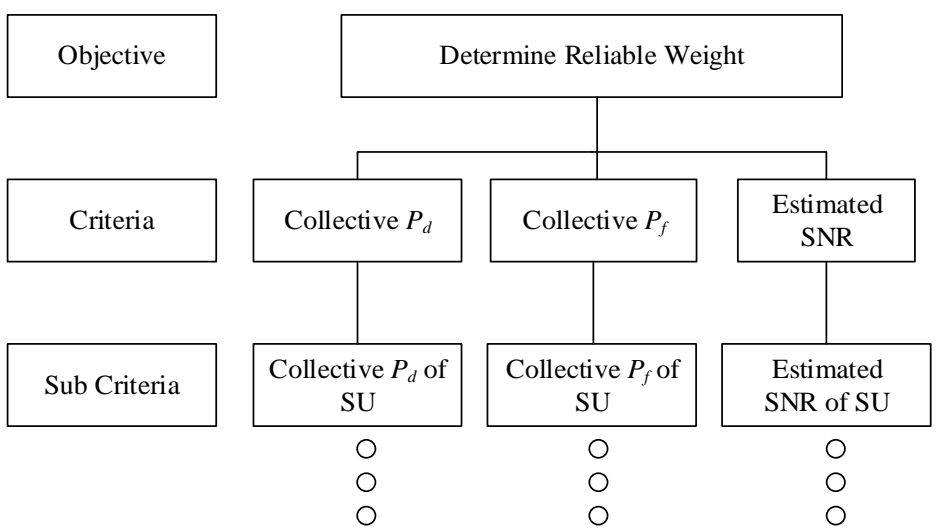

Figure 2. Structure of objective, criteria and sub-criteria.

\subsection{Adaptive AMT Thresholding}

In this sub-section, the AMT decision threshold $\left(\lambda_{\mathrm{AMT}}\right)$ and AMT decision statistic $\left(D_{\mathrm{AMT}}\right)$ are determined. Firstly, the optimal number of SU for AMT $\left(n_{\mathrm{AMT}}\right)$ is determined. As stated in $[43,44]$, the optimal number can be determined through the probability of false alarm $\left(Q_{f}\right)$ and the missed detection probability $\left(Q_{m}\right)$ of the majority rule where $Q_{m}$ can be given as:

$$
Q_{m}=\operatorname{Prob}\left\{H_{0} \mid H_{1}\right\}=1-\sum_{l=n}^{K_{R}}\left(\begin{array}{c}
K_{R} \\
l
\end{array}\right) P_{d}^{l}\left(1-P_{d}\right)^{K_{R}-l}
$$

Using (10) and (16), $n_{\text {AMT }}$ can be computed with an objective to minimize the error detection rate $\left(Q_{m}+Q_{f}\right)$. Given that $G$ is a function of the subtraction between $Q_{f}$ and $Q_{d}$ where $Q_{d}$ is in the term, $Q_{m}$ can be given as:

$$
Q_{d}=\operatorname{Prob}\left\{H_{1} \mid H_{1}\right\}=\sum_{l=n}^{K_{R}}\left(\begin{array}{c}
K_{R} \\
l
\end{array}\right) P_{m}^{K_{R}-l}\left(1-P_{m}\right)^{l}
$$


where $P_{m}$ is the local probability of missed detection of each SU.

Then, $G$ is given as:

$$
G(n)=\sum_{l=n}^{K_{R}}\left(\begin{array}{c}
K_{R} \\
l
\end{array}\right)\left[P_{f}^{l}\left(1-P_{f}\right)^{K_{R}-l}-P_{m}^{K_{R}-l}\left(1-P_{m}\right)^{l}\right]
$$

To obtain $n_{\mathrm{AMT}}, \frac{\partial G(n)}{\partial n}$ is 0 . Then

$$
\begin{gathered}
\frac{\partial G(n)}{\partial n} \approx G(n+1)-G(n) \\
0=\left(\begin{array}{c}
K_{R} \\
n
\end{array}\right)\left[\left(1-P_{m}\right)^{n} P_{m}^{K_{R}-n}-P_{f}^{n}\left(1-P_{f}\right)^{K_{R}-n}\right]
\end{gathered}
$$

where $P_{f}$ is the local probability of false alarm of each SU.

$$
P_{f}^{n}\left(1-P_{f}\right)^{K_{R}-n}=\left(1-P_{m}\right)^{n} P_{m}^{K_{R}-n}
$$

Given that

$$
\alpha_{\mathrm{AMT}}=\frac{\ln \frac{P_{f}}{1-P_{m}}}{\ln \frac{P_{m}}{1-P_{f}}}
$$

Then,

$$
n_{\mathrm{AMT}} \approx \frac{K_{R}}{1+\alpha_{\mathrm{AMT}}}
$$

It should be noted that the traditional majority rule set $\alpha_{\mathrm{AMT}}$ to 1 , then $n_{\mathrm{AMT}}$ is $\mathrm{N} / 2$. In our method, we determine the detection results from the $K_{R}$ of SUs that presents the summation of $\mathbf{W}_{\text {crs }}$ to $75 \%$. Therefore, $\lambda_{\text {AMT }}$ can be expressed as

$$
\lambda_{\mathrm{AMT}}=\left(\frac{n_{\mathrm{AMT}}}{K_{R}}\right) \sum_{i=1}^{K_{R}} w_{c r s, i}
$$

It should be mentioned that the value of $\mathbf{W}_{\text {crs }}$ affects the tradeoff between $Q_{d}$ and $Q_{f}$. If the $\mathbf{W}_{\mathrm{crs}}$ is set to $100 \%$, then all SUs are taken into account. On the other hand, if the $\mathbf{W}_{\text {crs }}$ is set lower than $50 \%$, a small number are taken into account which may present a high $Q_{f}$. Therefore, the $\mathbf{W}_{\mathrm{crs}}$ is selected as $75 \%$ which is averaged from $100 \%$ and $50 \%$. Then, the issue of the detection tradeoff can be addressed.

The decision statistic of AMT $\left(D_{\mathrm{AMT}}\right)$ can be expressed as:

$$
D_{\mathrm{AMT}}=\mathbf{W}_{\mathrm{crs} \in K_{R}}^{\mathrm{T}} C_{K_{R}}
$$

where $C_{K_{R}}$ is the decision binary from the SUs that is the member of $K_{R}$ highest reliability weight of SUs $\left(\mathbf{W}_{\mathrm{crs}}\right)$.

Finally, the PU state can be determined as:

$$
\text { PU state }= \begin{cases}\text { not exist } & : D_{\mathrm{AMT}}<\lambda_{\mathrm{AMT}} \\ \text { exist } & : D_{\mathrm{AMT}} \geq \lambda_{\mathrm{AMT}}\end{cases}
$$

\section{Simulation Results}

In this section, we simulate the performance of five cooperative spectrum sensing techniques-AND, OR, majority rule, improved weight algorithm (IMA) and adaptive majority rule-as compared to the proposed technique-adaptive multicriteria thresholding algorithm (AMT) under shadowing effect. The energy detection is considered as the local spectrum sensing of each unlicensed user (SU). The simulated network consists of 1 licensed user (PU) and 10 SUs (N). To evaluate the performance under shadowing effect, 
$25 \%$ of SUs are encountered by the shadowing where the strength is set to be 0 to $5 \mathrm{~dB}$. The PU signal, wireless microphone, is contaminated by additive white Gaussian noise (AWGN) [53], where the noise power is set to be $-96 \mathrm{dBm}$ as stated in $[54,55]$ and the signal-to-noise ratio (SNR) decreases on the path loss effect [12-14]. Then, the distance between PU and SUs is ranged from 10 to 500 meters $(\mathrm{m})$. Then, the SNR of wireless microphone signal decreases to $-23.46 \mathrm{~dB}$ at $500 \mathrm{~m}$. To evaluate the effect of SU's reliability, the distance of each SU is different from others in the range of $25 \%$ randomly.

In this evaluation, the two cooperative spectrum sensing performance metrics are considered including the probability of detection $\left(Q_{d}\right)$ and probability of false alarm $\left(Q_{f}\right)$. Moreover, we evaluate the robustness of cooperative spectrum sensing to shadowing effect from the percentage of shadowing robustness $(\mathrm{PB})$ which is given as:

$$
\mathrm{PB}=100-\left(\frac{\mid \text { Base } Q_{d}-\text { Shadowing }_{i \mathrm{~dB}} Q_{d} \mid}{\text { Base } Q_{d}}\right) \times 100
$$

where Base $Q_{d}$ is the $Q_{d}$ when shadowing is $0 \mathrm{~dB}$ and Shadowing $i \mathrm{~dB}$ is $Q_{d}$ when shadowing is $i \mathrm{~dB}$.

We investigate the performance of each cooperative spectrum sensing technique under different strengths of shadowing effect. As shown in Figure 3, the OR rule presents a high $Q_{d}$ for all distances. It can be noticed that the performance of detection of OR rule when shadowing is not taken into account is the highest as compared to the performance when shadowing is considered. As the cooperative spectrum sensing advantages, the detection performance decreases gradually when the strength of shadowing increases. Nevertheless, as depicted in Figure 4 , the false detection performance, $Q_{f}$, of OR rule is noticeably high while other techniques present low $Q_{f}$. Therefore, the OR rule cannot achieve the required performance of spectrum sensing where $Q_{d}$ should be as high as 0.9 and $Q_{f}$ should be as low as $0.1[55]$.



Figure 3. Investigation of the performance of $Q_{d}$ as a function of distance of the OR rule. 




Figure 4. Investigation of the performance of each cooperative spectrum sensing technique with comparison of $Q_{f}$ as a function of distance.

On the other hand, the other principle cooperative spectrum sensing technique, the AND rule, presents low $Q_{f}$ when PU does not actually exist. However, due to its detection strategy, the detection performance of the AND rule suffers from low SNR due to path loss and shadowing effect as shown in Figure 5a. It can be seen that the AND rule achieves spectrum sensing requirement at shadowing $0 \mathrm{~dB}$ when the distance is lower than $150 \mathrm{~m}$. Moreover, it achieves the requirement at a lower distance when the shadowing strength increases. Since a PU detection from the two principal strategies, OR and AND rule, cannot achieve the sensing performance on both metrics $\left(Q_{d}\right.$ and $\left.Q_{f}\right)$, the majority rule is encouraged to be used as the cooperative spectrum sensing. As shown in Figure 5b, the detection performance of the majority rule can achieve the spectrum sensing requirement when the distance is lower than $250 \mathrm{~m}$.

To improve the performance of traditional majority rule, the reliability of SU is taken into account. As depicted in Figure 5 c, the adaptive majority rule presents high $Q_{d}$ when distance is lower than $250 \mathrm{~m}$. It improves the robustness of shadowing performance of the majority rule since the adaptive majority rule. On the other hand, as shown in Figure $5 \mathrm{~d}$, IMA algorithm aims to minimize total error rate by computing the optimal required number of SUs and takes the reliability factor into account. As a result, IMA achieves high $Q_{d}$ for 1 to $3 \mathrm{~dB}$ of shadowing strength at $250 \mathrm{~m}$ which outperforms the detection performance of traditional majority and the adaptive majority rule. As depicted in the table, IMA algorithm shows a robustness to shadowing. As depicted in Figure 5e, by improving the performance of reliability weight computing, our proposed cooperative spectrum sensing, AMT, presents $Q_{d}$ higher 0.9 for all shadowing strengths at $250 \mathrm{~m}$. Table 1 presents the percentage of shadowing robustness $(\mathrm{PB})$ at 1 to $5 \mathrm{~dB}$ of four techniques as a function of distance at 250 . The $\mathrm{PB}$ results show that AMT gives the highest percentage of shadowing robustness (PB). 


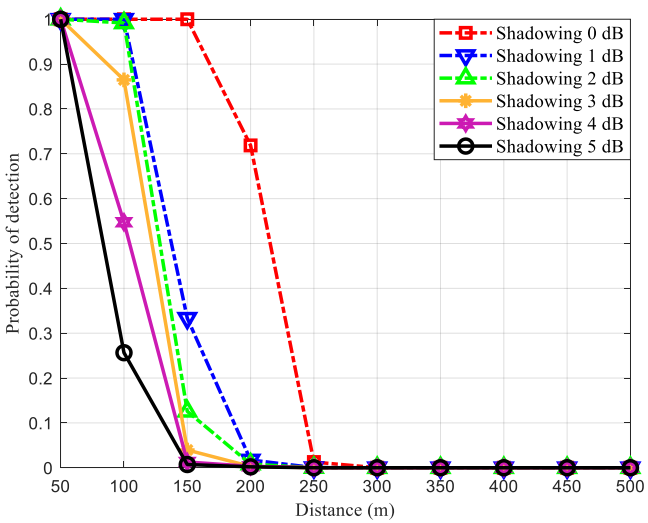

(a)

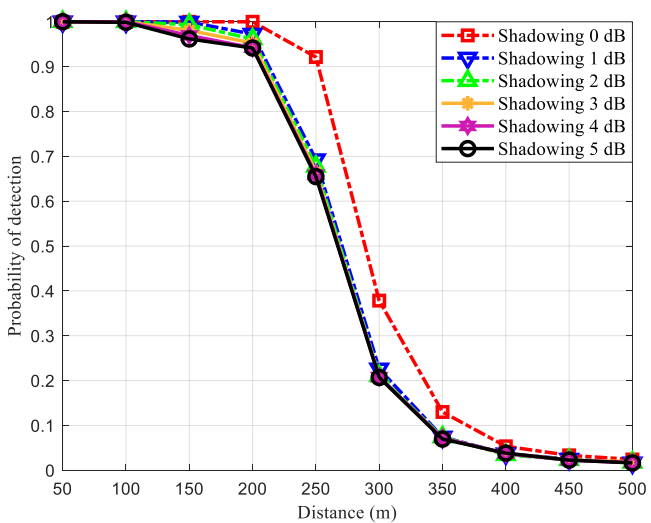

(c)

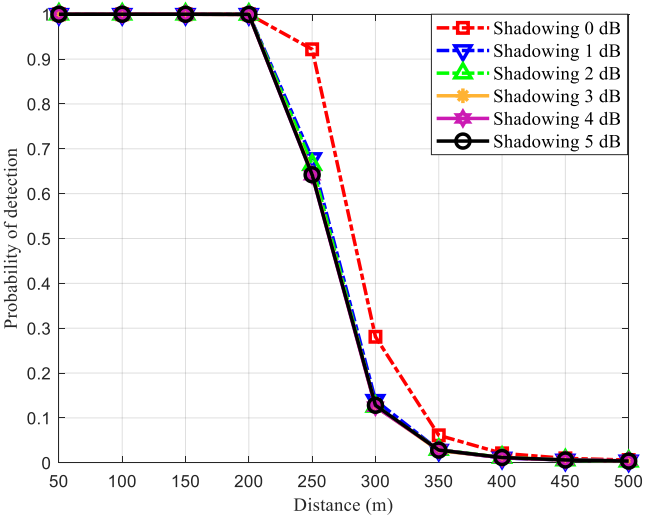

(b)

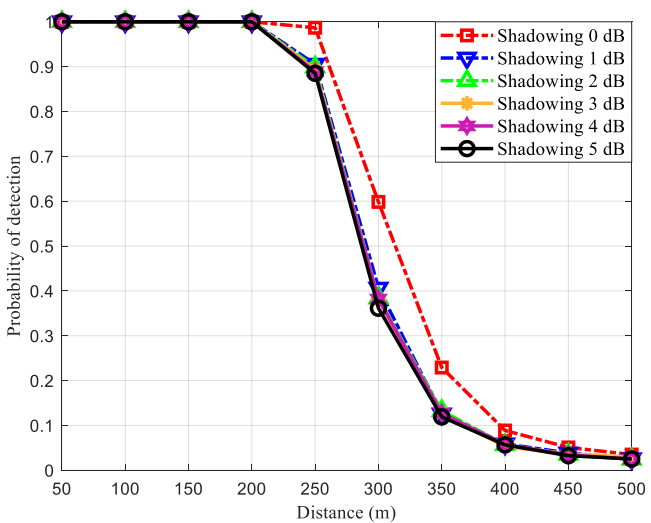

(d)

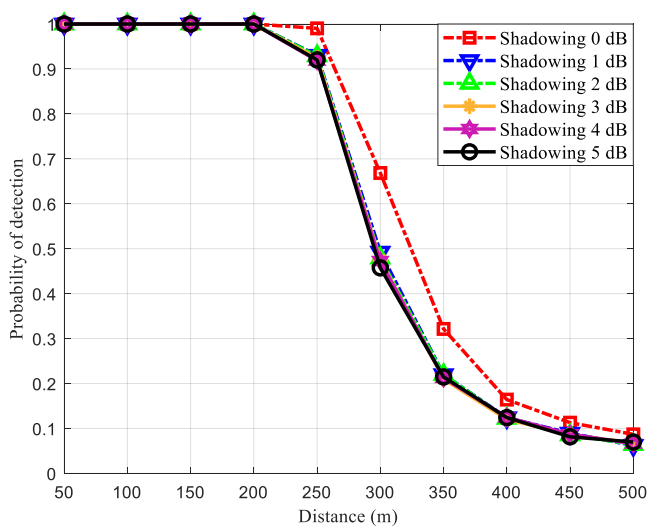

(e)

Figure 5. Investigation of $Q_{d}$ as a function of distance of each cooperative spectrum sensing technique. (a) AND rule; (b) the majority rule; (c) Adaptive majority rule; (d) IMA algorithm; (e) AMT algorithm.

Table 1. The percentage of shadowing robustness (PB) for the shadowing at 1 to $5 \mathrm{~dB}$ as a function of distance at $250 \mathrm{~m}$.

\begin{tabular}{cccccc}
\hline Methods & $\mathbf{1 ~ d B}$ & $\mathbf{2} \mathbf{d B}$ & $\mathbf{3 ~ d B}$ & $\mathbf{4} \mathbf{d B}$ & $\mathbf{5} \mathbf{d B}$ \\
\hline Majority & $73.59 \%$ & $72.08 \%$ & $70.02 \%$ & $69.54 \%$ & $69.66 \%$ \\
Adaptive & $75.18 \%$ & $73.55 \%$ & $72.19 \%$ & $71.51 \%$ & $71.07 \%$ \\
Majority & $91.77 \%$ & $91.12 \%$ & $90.58 \%$ & $90.10 \%$ & $89.70 \%$ \\
IMA & $93.94 \%$ & $93.82 \%$ & $93.32 \%$ & $92.75 \%$ & $92.91 \%$ \\
AMT & &
\end{tabular}


Then, we compare the robustness to shadowing effect through the proposed factor which is given in (27). As depicted in Figure 6, at $250 \mathrm{~m}$, AMT presents the highest percentage of shadowing robustness for all strengths of shadowing effect. It can be noticed that the AMT and IMA algorithms present a percentage of shadowing robustness higher than $90 \%$ for all shadowing strengths since the optimal number of SUs is considered. On the other hand, by taking only reliability through only detection performance of local SU, the adaptive majority rule cannot satisfy the cooperative spectrum sensing as AMT and IMA algorithm.

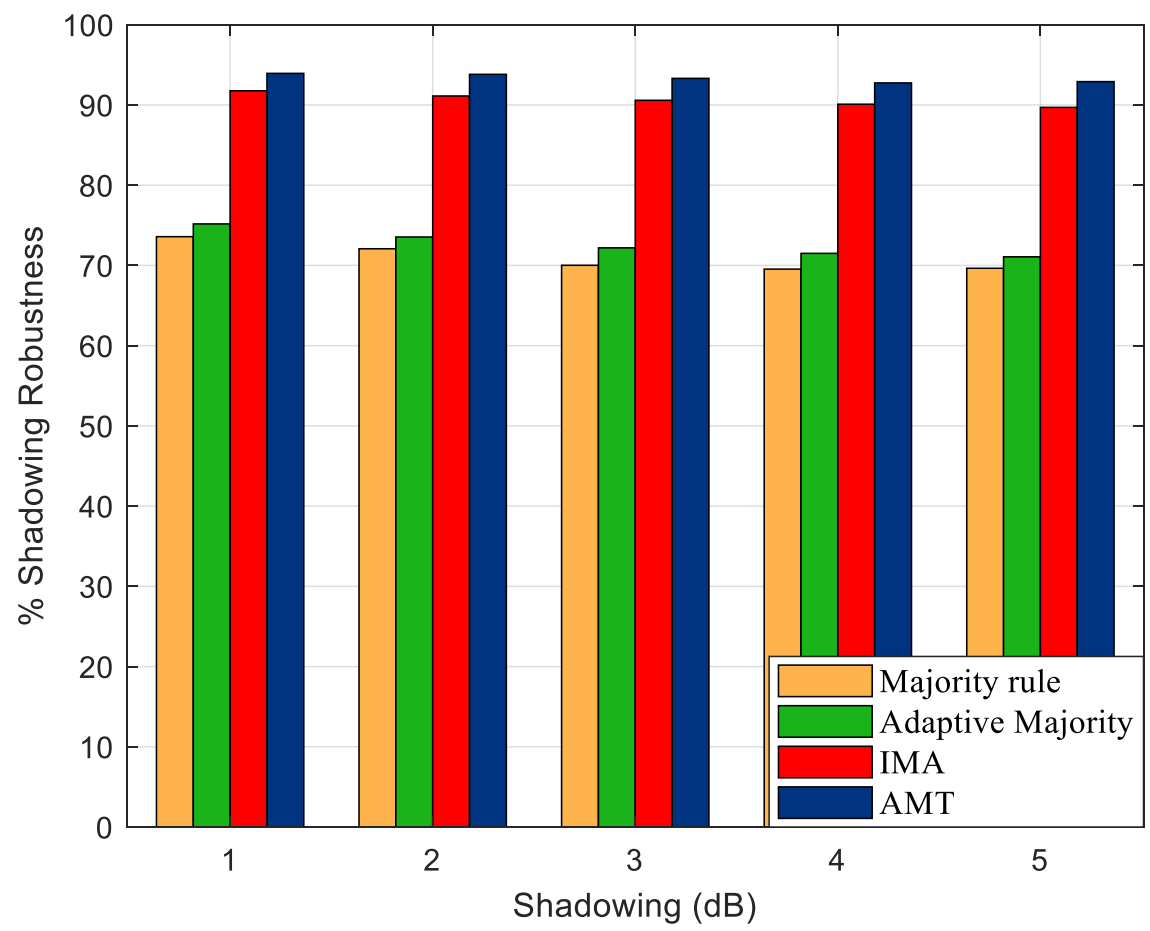

Figure 6. Comparison of the percentage of shadowing robustness at $250 \mathrm{~m}$.

From the simulation results, by taking the reliability of SUs into account, the adaptive majority rule, IMA and AMT algorithm improve the detection performance of the three traditional techniques-OR, AND and majority rule. Since the optimal number of SU is taken into account, both IMA and AMT outperform the performance of the adaptive majority rule. It can be seen that the threshold of IMA is according to the ratio between the optimal number of SU $\left(n_{\text {opt }}\right)$ and the actual number of SU $(N)$. The decision statistic of IMA can be determined as the linear combination between the weight vector and the local decision vector where the weight vector is according to the collective probability of detection and the local decision vector is the local decision from each SU. Although IMA determines the reliability of SUs on a single constrain and then it determines the channel availability according to the decision threshold, the IMA still takes the unreliable SUs into account which makes the detection performance suffer.

On the other hand, AMT determines the optimal number of SUs according to the highest reliable SUs where the reliability weights are calculated through analytic hierarchy process $(\mathrm{AHP})$ under three major performance metrics of spectrum sensing-collective detection rate, collective false detection rate and estimated SNR of detected signal. Therefore, the reliability of SUs can be determined comprehensively. Then, the unreliable SUs can be determined and can be neglected from the channel availability detection process. Therefore, the performance of AMT outperforms the detection performance of IMA and AMT presents the best shadowing robustness performance.

In addition, the rank reversal phenomenon is the critical issue of multi-criteria ranking methods which reverses the ranking of decision alternatives when a new alternative is 
taken into account or one of the alternatives is removed from the account. However, the environment of the ad-hoc network is determined where each SU node suffers from shadowing effect, path-loss effect and AWGN noise differently, therefore, the profile of the local SU differs from others. Moreover, the number decision alternative is static and there is no adding or removing the alternatives from the account. Then, the rank reversal does not affect the performance of the AMT under an ad-hoc network.

However, the limitation of the proposed algorithm is according to the simplest local spectrum sensing technique where the energy detection is considered. Therefore, the reliable detection from the local user is limited by the distance between the node and the primary user transmitter. If the alternative local spectrum sensing is considered, the performance of the proposed technique may be improved.

\section{Conclusions}

By performing the cooperative spectrum sensing from the intrinsic information of unlicensed users (SUs), the performance of the three principle cooperative spectrum sensing techniques-OR, AND and majority rule-degrades under shadowing effect since the local information from SUs may be unreliable. To overcome the performance of these principal techniques, the reliability of unlicensed users (SUs) is taken into account by adaptive majority rule and improved weight algorithm (IMA). However, the performance of these two techniques is not robust to the shadowing effect since the reliability of SUs is not considered comprehensively. In this paper, we propose a new cooperative spectrum sensing, adaptive multi-criteria thresholding (AMT), to address the shadowing issue of existing cooperative spectrum sensing techniques by exploiting the analytic hierarchy process (AHP). Once collective correct detection, collective false detection and estimated SNR of PU signal are taken into account, the reliability of SU can be determined efficiently. Moreover, the proposed decision threshold is determined by the number of reliable SUs and optimal number of SUs, therefore AMT presents the highest shadowing robustness performance as compared to others.

It could be mentioned that the proposed algorithm is evaluated only on a specific number of PUs. In the future work, we will evaluate the performance of the proposed technique with a various number of PUs. Moreover, the algorithm is based on the static PU whose location is fixed. Then, the only three considered criteria may not satisfy the spectrum sensing performance since the PU is considered as a mobile node. In this paper, we considered only one multi-criteria decision-making method. Then, we will take the mobile node environment with the criteria for the mobile node into account. In addition, the algorithm of multi-criteria decision making will be modified to address the mobile node issues.

Since the rank reversal can occur in practical environments in the form of duplicate profiles of alternatives, the case of duplicate profile may occur when the number of SU increases. Therefore, in the future work, we evaluate the performance of AMT when the number of SUs increases and investigate the effect of rank reversal. Moreover, the alternative solution will be presented to address the issue.

Author Contributions: Conceptualization, K.S. and Y.S.; methodology, K.S.; software, K.S.; validation, K.S., Y.S. and W.L.; formal analysis, Y.S.; investigation, K.S.; resources, W.L.; data curation, Y.S.; writing-original draft preparation, W.L.; writing—review and editing, Y.S.; visualization, W.L.; supervision, K.S.; project administration, W.L. All authors have read and agreed to the published version of the manuscript.

Funding: This research received no external funding.

Institutional Review Board Statement: Not applicable.

Informed Consent Statement: Not applicable.

Conflicts of Interest: The authors declare no conflict of interest. 


\section{References}

1. NIST Framework and Roadmap for Smart Grid Interoperability Standards, Release 3.0. Available online: http:/ / dx.doi.org/10.6 028/NIST.SP.1108r3 (accessed on 24 November 2020).

2. Wyglinski, A.M.; Nekovee, M.; Hou, Y.T. Cognitive Radio Communications and Networks: Principles and Practice; Elsevier Science Ltd.: Burlington, MA, USA, 2009.

3. Mitola, J.; Maguire, G.Q., Jr. Cognitive radio: Making software radios more personal. IEEE Pers. Commun. 1999, 6, 13-18. [CrossRef]

4. Akyildiz, I.F.; Lee, W.-Y.; Vuran, M.C.; Mohanty, S. NeXt generation/dynamic spectrum access/cognitive radio wireless networks: A survey. Comput. Netw. 2006, 50, 2127-2159. [CrossRef]

5. Akyildiz, I.F.; Lee, W.-Y.; Vuran, M.C.; Mohanty, S. A survey on spectrum management in cognitive radio networks. IEEE Commun. Mag. 2008, 46, 40-48. [CrossRef]

6. Wang, B.; Liu, K.R. Advances in cognitive radio networks: A survey. IEEE J. Sel. Top. Signal Process. 2011, 5, 5-23. [CrossRef]

7. Bisdikian, C. An overview of the Bluetooth wireless technology. IEEE Commun. Mag. 2001, 39, 86-94. [CrossRef]

8. Cordeiro, C.; Challapali, K.; Birrul, D. IEEE 802.22: An Introduction to the First Wireless Standard based on Cognitive Radios. J. Commun. 2006, 1, 38-47. [CrossRef]

9. Chowdhury, K.; Akyildiz, I. Cognitive Wireless Mesh Networks with Dynamic Spectrum Access. IEEE J. Sel. Areas Commun. 2008, 26, 168-181. [CrossRef]

10. Akyildiz, I.F.; Lee, W.-Y.; Chowdhury, K.R. CRAHNs: Cognitive radio ad hoc networks. AD Hoc Netw. 2009, 7, 810-836. [CrossRef]

11. Yucek, T.; Arslan, H. A Survey of Spectrum Sensing Algorithmsfor Cognitive Radio Applications. IEEE Commun. Surv. Tutor. 2009, 11, 116-130. [CrossRef]

12. Chenxi, L.; Miao, Y. A Distance-Weighed Algorithm Based on Maximum-Minimum Eigenvalues for Cooperative Spectrum Sensing. In Proceedings of the International Conference on Wireless Communications, Networking and Mobile Computing, Wuhan, China, 23-25 September 2011; pp. 1-4.

13. Zarin, N.; Mahmud, S.A.; Khan, I. Relay based cooperative spectrum sensing in cognitive radio networks over rayleigh fad-ing channel with path loss effects. In Proceedings of the IEEE 15th International Multi Topic Conference, Islamabad, Pakistan, 13-15 December 2012; pp. 291-296.

14. Faruk, N.; Ayeni, A.; Adediran, Y.A. On the study of empirical path loss models for accurate prediction of tv signal for sec-ondary users. Prog. Electromagn. Res. B 2013, 49, 155-176. [CrossRef]

15. Lopez-Benitez, M.; Casadevall, F. Signal Uncertainty in Spectrum Sensing for Cognitive Radio. IEEE Trans. Commun. 2013, 61, 1231-1241. [CrossRef]

16. Zhang, W.; Mallik, R.K.; Ben Letaief, K. Optimization of cooperative spectrum sensing with energy detection in cognitive radio networks. IEEE Trans. Wirel. Commun. 2009, 8, 5761-5766. [CrossRef]

17. Nallagonda, S.; Roy, S.D.; Kundu, S. Performance of Cooperative Spectrum Sensing in Log-normal Shadowing and Fading under Fusion Rules. Int. J. Energy Inf. Commun. 2012, 3, 15-28.

18. Subhedar, M.; Birajdar, G. Spectrum Sensing Techniques in Cognitive Radio Networks: A Survey. Int. J. Next-Gener. Netw. 2011, 3 , 37-51. [CrossRef]

19. Lu, L.; Zhou, X.; Onunkwo, U.; Li, G.Y. Ten years of research in spectrum sensing and sharing in cognitive radio. EURASIP J. Wirel. Commun. Netw. 2012, 2012, 28. [CrossRef]

20. Zeng, Y.; Liang, Y.-C.; Hoang, A.T.; Zhang, R. A Review on Spectrum Sensing for Cognitive Radio: Challenges and Solutions. EURASIP J. Adv. Signal Process. 2010, 2010, 381465. [CrossRef]

21. Malik, S.A.; Shah, M.; Dar, A.; Haq, A.; Khan, A.; Javed, T.; Khan, S.A. Comparative Analysis of Primary Transmitter Detec-tion Based Spectru Sensing Techniques in Cognitive Radio Systems. Aust. J. Basic Appl. Sci. 2010, 4, 4522-4531.

22. Mate, A.; Lee, K.-H.; Lu, I.-T. Spectrum sensing based on time covariance matrix using GNU radio and USRP for cognitive radio. In Proceedings of the 2011 IEEE Long Island Systems, Applications and Technology Conference, Farmingdale, NY, USA, 6 May 2011; pp. 1-6.

23. Nair, P.R. A Fast Sensing Algorithm for Spectrum Detection in Cognitive Radios. Master's Thesis, Nanyang Technological Uni-versity, Singapore, 2012.

24. Nair, P.R.; Vinod, A.P.; Krishna, A.K. An Adaptive Threshold Based Energy Detector for Spectrum Sensing in Cognitive Ra-dios at Low SNR. In Proceedings of the International Conference ICCS, Singapore, 17-19 November 2010; pp. 574-578.

25. Gorcin, A.; Qaraqe, K.A.; Celebi, H.; Arslan, H. An Adaptive Threshold Method for Spectrum Sensing in Multi-Channel Cognitive Radio Networks. In Proceedings of the 17th International Conference ICT, Doha, Qatar, 4-7 April 2015; pp. 425-429.

26. Li, Z.; Wang, H.; Kuang, J. A two-step spectrum sensing scheme for cognitive radio networks. In Proceedings of the International Conference on Information Science and Technology, Nanjing, China, 26-28 March 2011; pp. 694-698.

27. Srisomboon, K.; Prayote, A.; Lee, W. Multi-slot Double Constraints Adaptive Energy Detection for Spectrum Sensing in Cog-nitive Radio Networks. In Proceedings of the IEEE International Symposium on Communications and Information Technology, Nara, Japan, 7-9 October 2015.

28. Zeng, Y.; Liang, Y. Maximum-Minimum Eigenvalue Detection Forcognitive Radio. In Proceedings of the 2007 IEEE 18th International Symposium on Personal, Indoor and Mobile Radio Communications, Athens, Greece, 3-7 September 2007; pp. 1-5. 
29. Teshome, A.T. FPGA-Based Eigenvalue Detection Algorithm for Cognitive Radio. Master's Thesis, University of Gävle, Gävle, Sweden, 2010.

30. Ejaz, W.; Hasan, N.U.; Azam, M.A.; Kim, H.S. Improved local spectrum sensing for cognitive radio networks. EURASIP J. Adv. Signal Process. 2012, 2012, 242. [CrossRef]

31. Zhang, P.; Qiu, R. GLRT-Based Spectrum Sensing with Blindly Learned Feature under Rank-1 Assumption. IEEE Trans. Commun. 2012, 61, 87-96. [CrossRef]

32. Zhang, P.; Qiu, R.; Guo, N. Demonstration of Spectrum Sensing with Blindly Learned Features. IEEE Commun. Lett. 2011, 15, 548-550. [CrossRef]

33. Zhang, P.; Qiu, R. Spectrum Sensing Based on Blindly Learned Signal Feature. arXiv 2011, arXiv:1102.2840.

34. Lee, W.; Srisomboon, K.; Prayote, A. Fast Spectrum Sensing with Coordinate System in Cognitive Radio Networks. ETRI J. 2015, 37, 491-501. [CrossRef]

35. Ghasemi, A.; Sousa, E.S. Collaborative Spectrum Sensing for Opportunistic Access in Fading Enviroments. In Proceedings of the IEEE Symp. New Frotiers in Dynamic Spectrum Access Networks, Baltimore, MD, USA, 8-11 November 2005; pp. 131-136.

36. Tanu; Kaur, D. A Solution to the Hidden Node Problem in Cognitive Radio Networks. In Proceedings of the 4th IEEE International Conference on Signal Processing, Computing and Control (ISPCC 2k17), Solan, India, 21-23 September 2017.

37. Teguig, D.; Scheers, B.; Le Nir, V. Throughput Optimization for Cooperative Spectrum Sensing in Cognitive Radio Networks. In Proceedings of the 2013 Seventh International Conference on Next Generation Mobile Apps, Services and Technologies; Institute of Electrical and Electronics Engineers (IEEE), Prague, Czech Republic, 25-27 September 2013; pp. $237-243$.

38. Meena, O.P.; Somkuwar, A. Comparative Analysis of Information Fusion Techniques for Cooperative Spectrum Sensing in Cognitive Radio Networks. In Proceedings of International Conference on Recent Trends in Information, Telecommunication and Computing, ITC; Association of Computer Electronics and Electrical Engineers: Chandigarh, India, 2014; pp. $136-143$.

39. Atapattu, S.; Tellambura, C.; Jiang, H. Energy Detection Based Cooperative Spectrum Sensing in Cognitive Radio Networks. IEEE Trans. Wirel. Commun. 2011, 10, 1232-1241. [CrossRef]

40. Teguig, D.; Scheers, B.; Nir, V.L. Data Fusion Schemes for Cooperative Spectrum Sensing in Cognitive Radio Networks. In Proceedings of the Communications and Information Systems Conference (MCC), Gdansk, Poland, 8-9 October 2012.

41. Do, N.T.; An, B. A Soft-Hard Combination-Based Cooperative Spectrum Sensing Scheme for Cognitive Radio Networks. Sensors 2015, 15, 4388-4407. [CrossRef] [PubMed]

42. Gupta, M.; Verma, G. Improved Weighted Cooperative Spectrum Sensing Algorithm Based on Reliability in Cognitive Radio Networks. In Proceedings of the IEEE International Conference on Recent Trends in Electronics Information Communication Technology, Bangalore, India, 20-21 May 2016; pp. 609-612.

43. Liang, Y.C.; Zeng, Y.; Peh, C.Y.E.; Hoang, A.T. Sensing-Throughput Tradeoff for Cognitive Radio Networks. IEEE Trans. Wirel. Commun. 2008, 7, 1326-1337. [CrossRef]

44. Akyildiz, I.F.; Lo, B.F.; Balakrishnan, R. Cooperative spectrum sensing in cognitive radio networks: A survey. Phys. Commun. 2011, 4, 40-62. [CrossRef]

45. Srisomboon, K.; Sroulsrun, Y.; Lee, W. Adaptive Majority Rule in Cooperative Spectrum Sensing for Ad hoc Network. In Proceedings of the 2020 8th International Electrical Engineering Congress (iEECON), Chiang Mai, Thailand, 4-6 March 2020; pp. $1-4$.

46. Liu, X.; Zheng, K.; Chi, K.; Zhu, Y.-H. Cooperative Spectrum Sensing Optimization in Energy-Harvesting Cognitive Radio Networks. IEEE Trans. Wirel. Commun. 2020, 19, 7663-7676. [CrossRef]

47. Analytic Hierarchy Process. The Analytic Hierarchy Process; Computing: New York, NY, USA, 1980.

48. Saaty, W. The analytic hierarchy process-what it is and how it is used. Math. Model. 1987, 9, 161-176. [CrossRef]

49. Analytic Hierarchy Process (AHP) Tutorial. Available online: http://people.revoledu.com/kardi/tutorial/AHP/AHP.htm (accessed on 5 January 2020).

50. Zhou, X.; Sun, M.; Li, G.Y.; Juang, B.F. Intelligent wireless communications enabled by cognitive radio and machine learning. China Commun. 2018, 15, 16-48.

51. Cai, P.; Zhang, Y. Intelligent cognitive spectrum collaboration: Convergence of spectrum sensing, spectrum access, and cod-ing technology. Intell. Converg. Netw. 2020, 1, 1.

52. Salabun, W.; Watrobski, J.; Shekhovtsov, A. Are MCDA Methods Benchmarkable? A Comparative Study of TOPSIS, VIKOR, COPROS, and PROMETHEE II Methods. Symmetry 2020, 12, 1549. [CrossRef]

53. Additive White Gaussian Noise (AWGN). Available online: http://www-isl.stanford.edu/ \{\}cover/papers/transIT/0572cove. pdf (accessed on 11 March 2020).

54. Shellhammer, S.J. Sensitivity Requirement for Sensing Wireless Microphones. IEEE 802.22-07/0290r3 2007. Available online: https: //mentor.ieee.org/802.22/dcn/07/22-07-0290-03-0000-sensitivity-requirement-for-sensing-wireless-microphones.doc (accessed on 15 January 2020).

55. Chouinard, G. Sensing performance with the 802.22.1 wireless microphone beacon. IEEE 802.22-09/0068r1 2009. Available online: https: / / mentor.ieee.org/802.22/dcn/09/22-09-0068-01-0000-sensing-performance-from-802-22-1-wireless-microphonebeacon.doc (accessed on 15 January 2020). 